\title{
A FINITE BUFFER SCHEDULING SCHEME WITH STATISTICAL GUARANTEE FOR VBR TRAFFIC IN ATM NETWORKS *
}

\author{
Arthur M. O. Lai, Danny H.K. Tsang \\ Department of Electrical and Electronic Engineering \\ Hong Kong University of Science and Technology \\ Clear Water Bay, Kowloon, Hong Kong \\ email: (eearthur, eetsang)@ee.ust.hk
}

\begin{abstract}
Future ATM networks will support real-time voice and video services and we need to guarantee cell transfer delay and cell loss probability to launch such services. The Earliest Deadline First (EDF) scheme is a suitable service scheduling scheme for these services since it has been proven that it can minimize the number of late cells by assigning deadlines to the cells. However, the EDF scheme considers only the infinite buffer case and is susceptible to buffer overflow when finite buffer is considered. In this paper, we propose a novel scheme, Earliest Deadline First for Finite Buffer (EDFFB), which is able to provide a statistical delay bound while having a lower chance of buffers overflow than the EDF scheme.
\end{abstract}

\section{Introduction}

Service scheduling scheme is the mechanism that controls the order of servicing cells from different input streams within an ATM node. Proper choice of service scheduling scheme is very important in controlling the delay of ATM cells and preventing buffers overflow. Numerous service scheduling schemes have been proposed in the literature $[6,5,2,4,3,1]$. Among the proposed schemes, the Earliest Deadline First (EDF) service scheduling scheme [3] has received much attention in providing real-time services. The EDF scheme is a very effective sharing scheme and also possesses a desirable feature that has proven to be optimal in a sense that it minimizes the number of cells that violate the deadline under any traffic pattern. However, the EDF scheme does not consider the finite buffer case and may lead to excessive cell loss due to lack of buffer space. For example, there are

${ }^{*}$ This research was supported in part by the Hongkong Telecom Institute of Information Technology grant: HKTIIT 93/94.EG01. five input traffic (each having its own queue) in a system, and only one queue is full. Although there are still some buffer space in the other four queues, losses can still occur since the scheduling scheme does not know that one of the queue is full and needs service first. Therefore any new arrival on the full queue will be dropped even there are still buffer space in other queues.

Many applications such as video conferencing and compressed voice/video transmission can tolerate some losses without too much degradation in quality and therefore can be supported by statistical guarantee to improve the utilization of system resources. In this paper, we focus on statistical guarantee instead of an absolute one. That is, we concentrate on services that can tolerate a small amount of losses due to delayed transmission and cells dropped because of limited buffer space. Here we propose a novel service scheduling scheme, the Earliest Deadline First for Finite Buffer (EDF-FB) scheme, that provides a statistical QoS guarantee in a per-VC or per-flow queueing environment with finite buffer size. Our scheme is also based on the well-known Earliest Deadline First (EDF). By incorporating buffer occupancy information to avoid buffer overflow in our scheme, we can provide a statistical QoS guarantee with a lower cell loss rate compared with the EDF scheme. The organization of the paper is as follows: In section 2, we describe our proposed scheduling scheme in detail. In section 3 , we provide the simulations results and discussions. We give the conclusion in section 4 .

\section{EDF-FB Scheme}

\subsection{System Model}

Consider the queueing system model as shown in Figure 1. There are $N$ sources accessing the ATM switch/multiplexer. All the sources are assumed to be properly policed by leaky bucket filters and 
therefore conform to their contracts. All the cells have the same fixed size. The leaky bucket filters are characterized by the parameters $(r, b)$, where $r$ denotes the token generation rate and $b$ denotes the token pool size. The QoS parameters specified are the maximum cell delay $\left(d_{i}\right)$ and the cell loss probability $\left(\epsilon_{i}\right)$. A cell is discarded when it is delayed over the pre-specified deadline or the buffer is full upon its arrival.

\subsection{The EDF-FB Algorithm}

The Earliest Deadline First for Finite Buffer (EDFFB) service scheduling scheme is designed to obtain a good delay performance while attempting to avoid overflow of buffers. The novel part of this scheme is that two non-simultaneous servers are used to determine the order of service for the cells. The EDF server tries to give an optimal delay performance for the system, and at the same time, the overflow protection (OP) server tries to avoid overflow of buffers.

The basic operation of EDF-FB is illustrated in the pseudo-code of Figure 2. The meanings of the variables used are provided in Table 1 to help the reader to understand the flow of the proposed algorithm. In the EDF-FB service scheduling scheme, cells from each source are queued in a separate buffer. In addition, a threshold is set for each queue to determine whether the queue is in danger of overflow or not. On the arrival of each cell, it will be assigned a time-stamp with a value equal to the current time plus the pre-specified deadline. At the beginning of each time slot, all the headof-line (HOL) cells will be checked first. If a cell has already been delayed beyond its deadline, it will be discarded and the next cell from the same queue will be promoted to the head of the queue and checked again and so on until a cell that does not violate its deadline is found. Under normal circumstances, the EDF-FB scheme works just like the EDF scheme. However, when the occupancy of one or more queues is above the threshold, the OP server will override the function of the EDF server. In this case, the OP server will compare the queue length of those queues with occupancy above their individual thresholds, and the HOL cell from the longest queue will be picked and served. In this way, those queues that are in danger of overflow will get service first and therefore be able to recover from the temporary overloading quickly. Our scheme is also work-conserving since a cell will always be picked and served if any one of the queues is backlogged. The server will only be idle if all the queues are empty.

The implementation of the EDF-FB scheme is

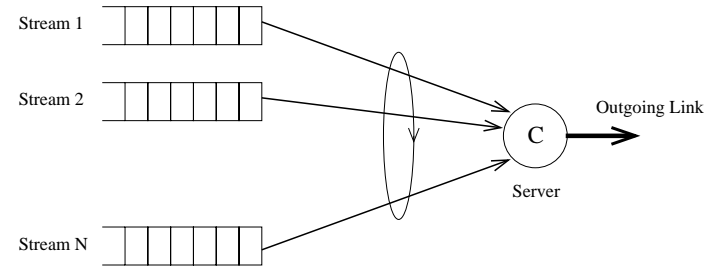

Figure 1: Queue System Model

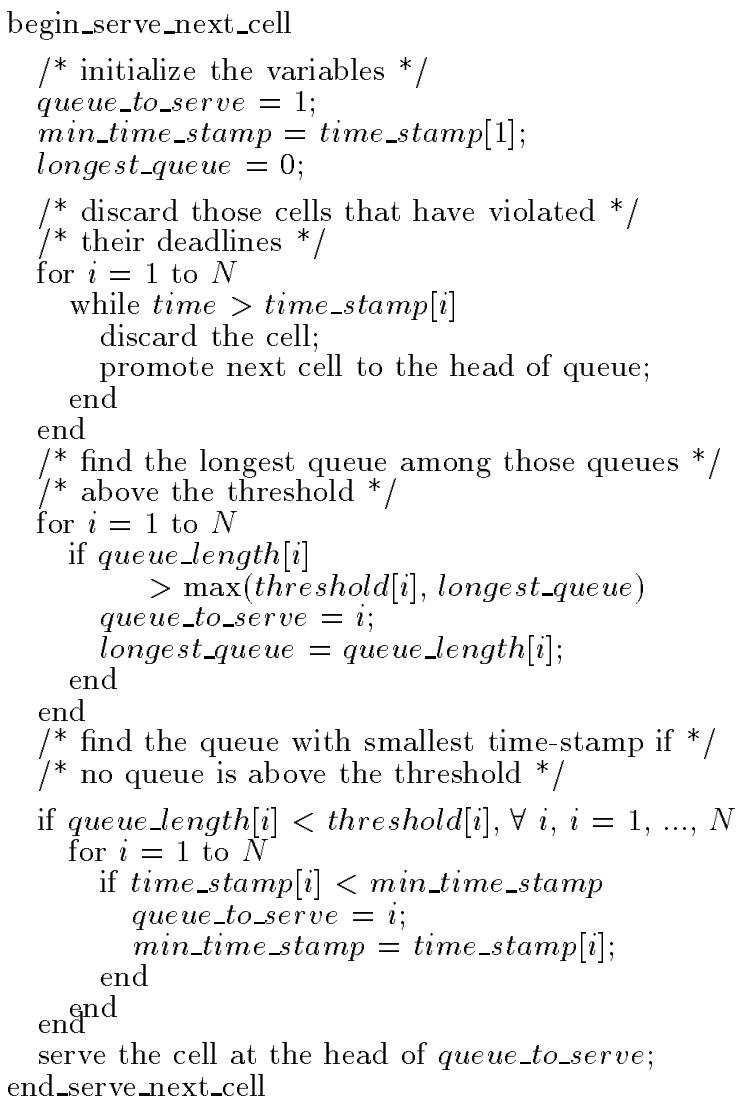

Figure 2: Pseudo-code for EDF-FB

\begin{tabular}{||l|l||}
\hline \hline Variable & Meaning \\
\hline$N$ & number of queues in the system \\
time & current time \\
time_stamp $[i]$ & time-stamp of HOL of queue $i$ \\
queue_length $[i]$ & queue length of queue $i$ \\
threshold $[i]$ & threshold value of queue $i$ \\
queue_to_serve & queue to be picked for service \\
longest_queue & length of the longest queue \\
min_time_stamp & the minimum time-stamp value \\
& of all the HOL cells \\
\hline \hline
\end{tabular}

Table 1: Meanings of variables 
simple. Any proposed implementation for the EDF scheme can be used as the EDF server in the EDFFB scheme. For queue length information, only a simple counter to keep track of the input and output for each queue is needed. At the beginning of each time slot, we only need to compare either the time-stamps of the HOL cells or the queue lengths. Comparison operations are generally needed in most of the service schemes. Therefore, introducing the OP server simply adds a minimal overhead to the system.

\section{Simulation Results}

In this section, we evaluate the performance of the EDF-FB scheme and compare it with the EDF scheme under different traffic conditions using discrete-time simulations (where each time slot represents the service time for a cell). The sources of real-time traffic are modelled as twostate Markov processes. In each burst period, a geometrically distributed random number of cells are generated at some peak rate $P$ and the average burst size is $B$. After the burst has been generated, the source remains idle for some exponentially distributed random time with $I$ denoting the average length of an idle period. The average rate of cell generation $A$ is given by $A^{-1}=\frac{I}{B}+\frac{1}{P}$. Each traffic source is subjected to an $(r, b)$ leaky bucket filter, where $r$ denotes the token generation rate and $b$ denotes the token pool size. Any cell passes through the filter will consume a token and a cell can only pass through the filter only if there are tokens in the token pool. Otherwise, it is considered to be nonconforming and will be dropped. In our simulations, 10 sources are connected to an outgoing link, which has bandwidth of $1000 \mathrm{cell} / \mathrm{s}$.

In section 3.1 , the mean burst size $B$ is set to 20 cells, the peak cell rate $P$ is 400 cells $/ \mathrm{s}$, and the mean idle period $I$ is $0.2 s$. The threshold is expressed in term of fraction of the buffer size and will be varied between 0 to 1 . In sections 3.2 and 3.3. We will fix the mean burst size to $20 \mathrm{cells}$, but the mean idle period and the peak cell rate are then changed so as to adjust the loading. The leaky bucket filter parameters are set as follows. The token generation rate is equal to the average cell generation rate and the token pool size is equal to ten times the average burst size.

\subsection{Effect of Threshold Setting}

In this section, we study how the threshold settings of the queues affect the delay and cell loss performance. Here all the sources are statistically identical with the same delay bound of $300 \mathrm{~ms}$. The effect

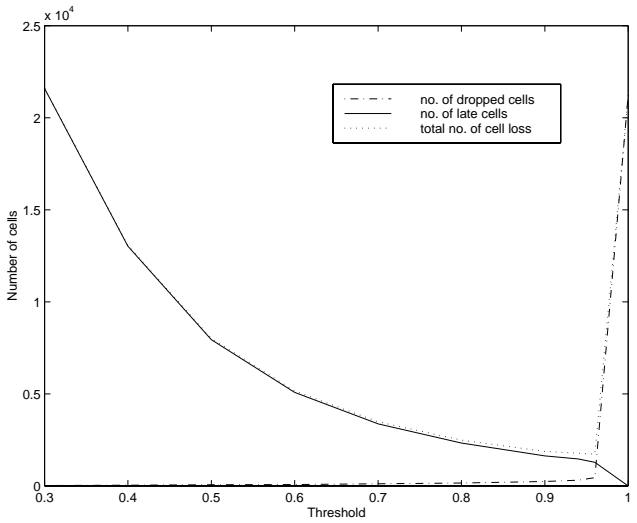

Figure 3: Cell Loss vs. Threshold

of changing the threshold is shown in Figure 3. If the threshold is small, the OP server will override the function of the EDF server for most of the time. Therefore the chance of buffer overflow is minimized but the delay will not be under control. As a result, cell loss due to lack of buffer space is small but a lot of cells are discarded due to deadline violation. When the threshold increases, the loss due to buffer overflow increases but at the same time the number of late cells decreases as more cells are scheduled by the EDF server. When the threshold is set to one, the EDF-FB scheme becomes the same as the EDF scheme and all the cell loss mainly comes from buffer overflow. One important observation is that the number of late cells decreases gradually with the increase of threshold values. On the other hand, the number of cells dropped due to lack of buffer space is small for threshold values between 0 and 0.95 but it increases very abruptly when the threshold is near one. This is because if the threshold is smaller than one, the OP server will come into action and those queues that are in danger of overflow will get service first. Further lowering the threshold does not help because the OP server is only needed when the queue is in danger of overflow. Consequently, there exists a threshold setting at which the total cell loss is minimum and this optimal threshold setting is usually close to one. We have therefore chosen the threshold setting to be 0.9 in the rest of our simulations. The optimal threshold may change slightly with the loading or buffer size; however, the method of obtaining this optimal threshold is subject to further research.

\subsection{Effect of Loading on Buffer Re- quirements}

In this section, we investigate the buffer requirements under different loading. In particular, we 
study the buffer size required under different loading for satisfying a given cell loss probability. The result is shown in Figure 4.

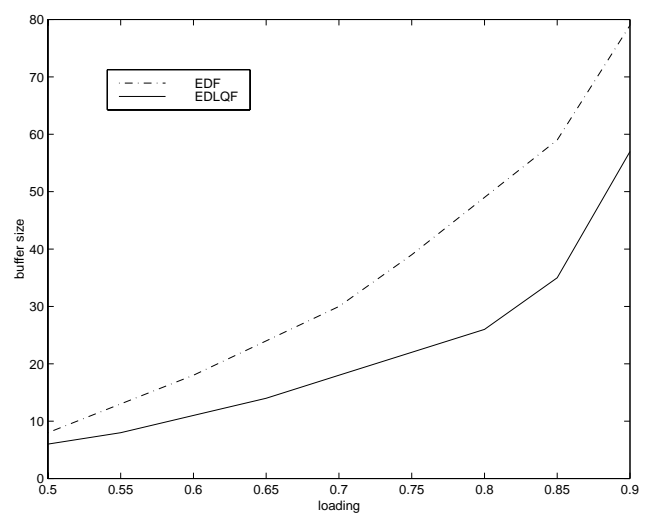

Figure 4: Buffer Size vs. Loading $\left(\epsilon=10^{-3}, d=\right.$ $300 \mathrm{~ms}$ )

As shown in the figure, the EDF-FB scheme always requires smaller buffer size than the EDF scheme to achieve the same cell loss probability. When the loading is low, the improvement of the EDF-FB scheme over the EDF scheme is relatively small. However, the improvement increases as the loading increases and a maximum improvement is obtained at the loading of around 0.8 . The improvement decreases when the loading is very heavy (over 0.9). The reason is that buffers are not likely to overflow when the loading is low. As the loading increases, there is a higher chance that some of the queues are being overloaded temporary, while other queues still have excess resources (both buffer and bandwidth) to help the overloading queues recover from the temporary overloading and avoid buffers overflow. However, when the loading becomes too heavy, all the queues are highly loaded and the EDF-FB scheme cannot help the overloading queues because now the system resources are the bottleneck. Since it is reasonable to assume that one should operate the system at neither low nor high loading, the EDF-FB scheme can be advantageous most of the time since it achieves the largest improvement in the region where the system should normally be operated.

\subsection{Effect of Buffer Size on Cell Loss Probability}

\subsubsection{Homogeneous Traffic}

First, we study the performance of our algorithm under homogeneous traffic environment in which all data streams are statistically identical and have the same delay requirement of $300 \mathrm{~ms}$.

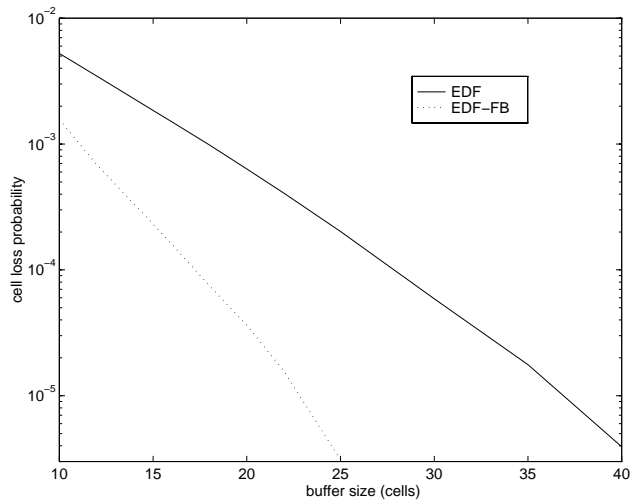

Figure 5: Cell Loss Probability vs. Buffer Size Under Moderate Loading and Homogeneous Traffic Condition (loading $=0.6, d=300 \mathrm{~ms}$ )

Under moderate loading, almost all the cells are schedulable under both schemes (i.e. all the cells can be served before their deadlines expire). Therefore, the cell loss mainly arises from cells dropped due to insufficient buffer space. From Figure 5, we can see that the EDF-FB scheme always outperforms the EDF scheme. When the buffer size is small, the cell loss probability of the EDFFB scheme is only slightly lower than that of the EDF scheme. This is because when the buffer size is very small, the buffer cannot hold all the incoming cells before they are served even all the bandwidth is given to the overloading queues. As the buffer size increases, the cell loss probability of the EDF-FB scheme decreases more quickly than that of the EDF scheme. In other words, the relative improvement of the EDF-FB scheme increases with the buffer size. As shown in Figure 5, the cell loss probability of the EDF-FB scheme is only a few times lower when the buffer size is 10 , but the cell loss probability of the EDF-FB scheme becomes lower by two orders of magnitude when the buffer size increases to 25 . This is because when the buffer is large, the buffer space can accommodate more cells during a burst and the OP server can help those overloading queues recover from temporary loading. Therefore the cell loss probability of the EDF-FB scheme decreases faster than that of the EDF scheme as the buffer size increases.

Next we investigate the effect of buffer size on cell loss probability under heavy loading. The result is shown in Figure 6. When the buffer increases, the cell loss probability first decreases very quickly. When the buffer size is up to about 35 , the improvement decreases as the buffer size increases further. The reason is similar to the moderate loading case when the buffer size is small. However, when the buffer size is large, the cells dis- 


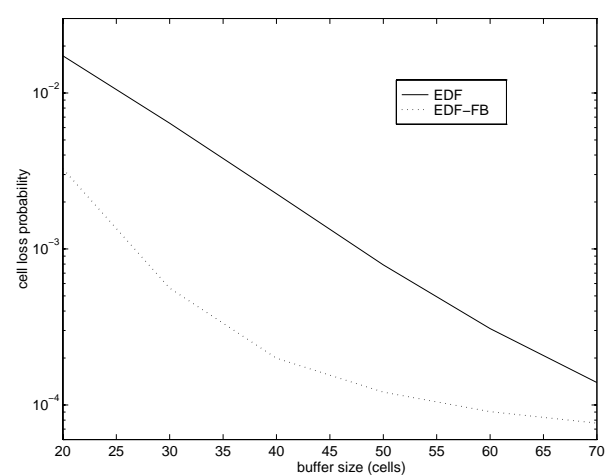

Figure 6: Cell Loss Probability vs. Buffer Size Under Heavy Loading and Homogeneous Traffic Condition (loading $=0.8, d=300 \mathrm{~ms}$ )

carded due to late transmission become dominant while the cell loss due to buffer overflow decreases. At the same time, both scheduling schemes cannot serve all the cells before their deadlines in this case. Although the EDF-FB scheme can reduce the cell loss due to lack of buffer space, it cannot improve the cell loss due to deadline violation. Therefore the relative improvement decreases as the buffer size increases. As it can be seen in the figure, there exists some optimal buffer size at which the EDFFB scheme achieves the highest relative improvement over the EDF scheme. This optimal point occurs when the EDF-FB scheme can continue to reduce the cell loss due to buffers overflow while the cells loss due to deadline violation is still not dominant.

\subsubsection{Heterogeneous Traffic}

The heterogeneous traffic environment is also considered. Here we keep the total number of sources to be 10 but they have different delay requirements. Five of them have a delay bound of $250 \mathrm{~ms}$ $(d=250 \mathrm{~ms})$ while the remaining five have a delay bound of $300 \mathrm{~ms}(d=300 \mathrm{~ms})$. We refer to those sources with $d$ of $250 \mathrm{~ms}$ as class 1 and those with $d$ of $300 \mathrm{~ms}$ as class 2 .

In Figure 7(a), the individual cell loss probabilities of the two classes are shown under different buffer sizes at moderate loading. For the EDF scheme, class 2 traffic always has a much higher cell loss probability than class 1 traffic. This implies that the cells with larger delay bound will be penalized under the EDF scheme because those cells with larger delay bound need to queue up and wait for a relatively longer time before they are served and are thus susceptible to buffer overflow. This is not desirable since those services that
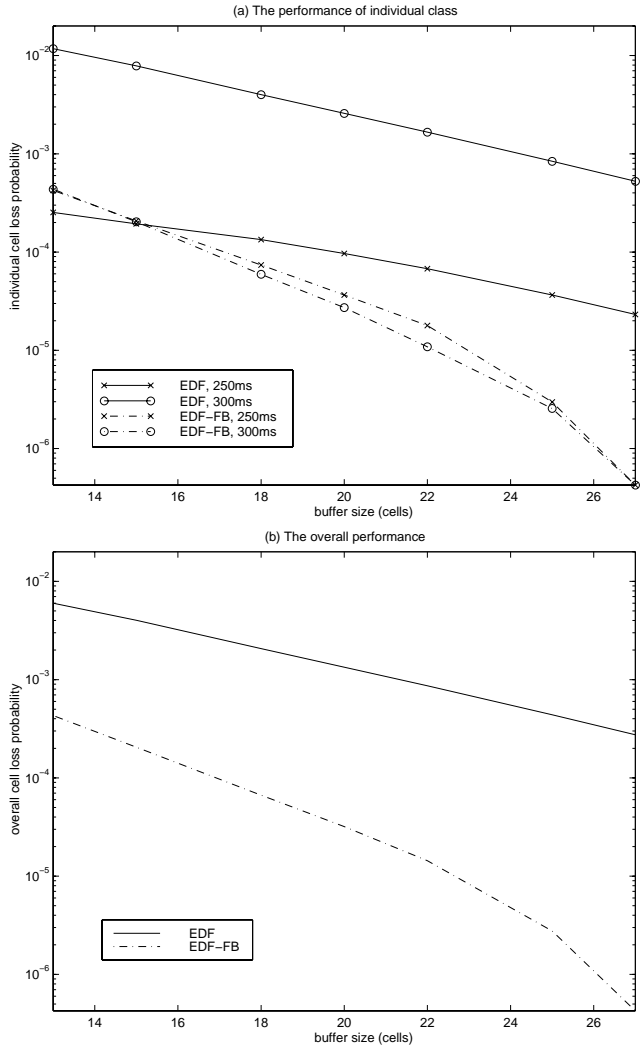

Figure 7: Cell Loss Probability vs. Buffer Size Under Moderate Loading and Heterogeneous Traffic Condition (loading $=0.6$ )

can tolerate a longer delay perhaps cannot tolerate a higher cell loss. On the other hand, in the EDF-FB scheme, the cell loss probabilities of the two classes are much closer to each other. This is because class 2 traffic can gain priority over class 1 traffic once its queues are built up above the threshold and thus the chance of buffers overflow is much reduced. In addition, except for very small buffer size, the cell loss probabilities of the EDF-FB scheme are smaller compared to the EDF scheme for both classes. The improvement is very significant for class 2 traffic since it has been penalized under the EDF scheme. In Figure 7(b), the overall cell loss probability of the two classes is shown. The EDF-FB scheme clearly outperforms the EDF scheme under different buffer sizes. The behaviour is very similar to the homogeneous case. The cell loss probability decreases much more quickly for the EDF-FB scheme and thus a much smaller buffer is needed for the EDF-FB scheme to achieve the same overall cell loss probability as the buffer size increases.

We now proceed to the heavy loading case as shown in Figure 8. As in the moderate loading case, class 2 traffic has a much higher cell loss 

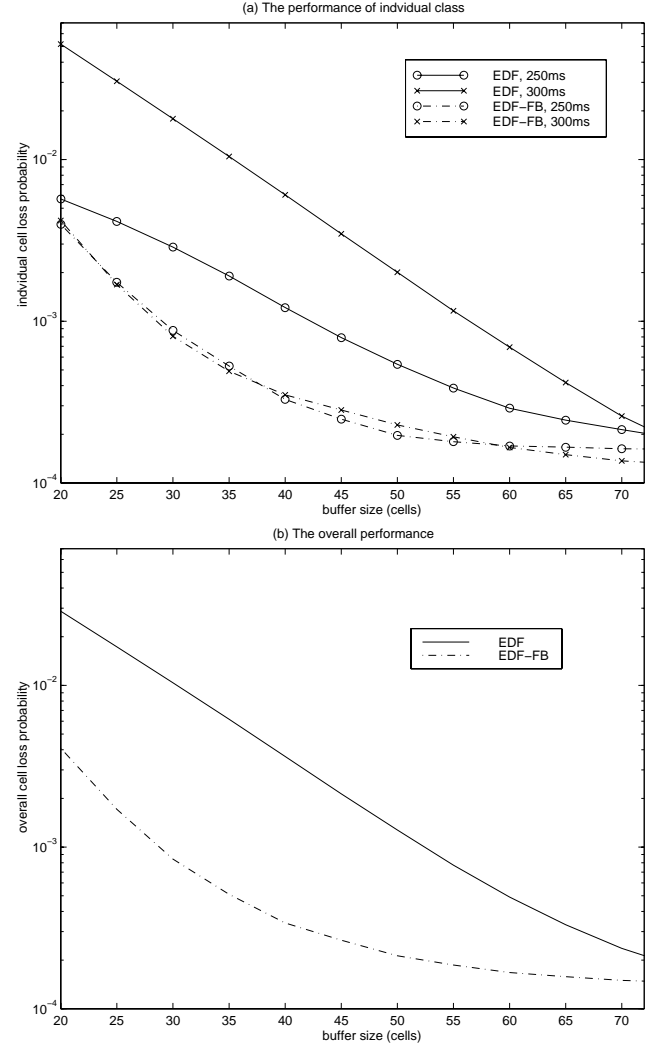

Figure 8: Cell Loss Probability vs. Buffer Size Under Heavy Loading and Heterogeneous Traffic Condition (loading $=0.8$ )

probability than class 1 traffic in the EDF scheme. In the EDF-FB scheme, both classes achieve a much lower cell loss probability and the cell loss probabilities of the two classes are very close to each other. The EDF-FB scheme results in a significant improvement (approximately an order of magnitude) for class 2 traffic while keeping the cell loss probability of class 1 traffic lower than that under the EDF scheme. The reason is the same as in the moderate loading case, the EDF-FB scheme provides a higher priority to class 2 traffic when their queue lengths are above the thresholds and this reduces the chance of buffers overflow. When the overall cell loss probability is considered, the EDF-FB scheme again outperforms the EDF scheme. Similar to the homogeneous case, the improvement of the EDF-FB scheme increases with buffer size when the buffer size is not too large. However, when the buffer size increases up to around 40 and 50, the improvement begins to decrease. This is because when the buffer size is small, most of the cell loss comes from buffer overflow and thus the EDF-FB scheme is very effective in reducing the loss. As the buffer size increases further, the number of late cells starts to dominate and the improvement of EDF-FB is thus reduced.

\section{Conclusions}

Numerous service scheduling algorithms have been proposed to guarantee QoS in ATM networks. These schemes can guarantee delay bounds but the finite buffer requirements have been ignored. In this paper, we have proposed a simple but effective service scheduling algorithm, Earliest Deadline First for Finite Buffer (EDF-FB), that provides a statistical QoS guarantee with a lower buffer requirement. The EDF-FB scheme which is based on the Earliest Deadline First (EDF) scheme and a overflow protection (OP) server is introduced to prevent buffer overflow. It attempts to control the number of cells that violate the deadline and at the same time to reduce the cell loss due to buffer overflow. Through simulations, we have shown that for a given buffer size, the EDF-FB scheme can achieve a much lower cell loss probability than the EDF scheme. In addition, we observe a significant improvement in buffer requirement over the normal operating region of networks.

\section{References}

[1] A.K. Parekh, R.G. Gallagher. A Generalized Processor Sharing Approach to Flow Control in Integrated Services Networks. in Proceedings of IEEE INFOCOMM'94.

[2] D.S. Lee. Weighted Longest Queue First: A Adaptive Scheduling Discipline for ATM Networks. Proceedings of IEEE INFOCOM'97, 1997.

[3] H. Saito. Optimal Queueing Discipline for Real-Time Traffic at ATM Switching Nodes. IEEE Transactions on Communications, Vol. 38, No. 12, Dec. 1990.

[4] J.C.R. Bennett, H. Zhang. $W F^{2} Q$ : Worstcase Fair Weighted Fair Queueing. Proceedings of IEEE INFOCOM'96, pp. 120-128, 1996.

[5] P. Goyal, H.M. Vin and H. Chen. Starttime Fair Queueing: A Scheduling Algorithm for Integrated Services Packet Switching Networks. Proceedings ACM SIGCOMM'96, pp.157-169.

[6] S.J. Golestani. A Self-Clocked Fair Queueing Scheme for Broadband Applications. Proceedings of INFOCOM'94, pp.636-646, 1994. 